PUBLICATIONS DE L'INSTITUT MATHÉMATIQUE

Nouvelle série, tome 75(89) (2004), 185-197

\title{
ON THE ZEROS OF FUNCTIONS IN THE BERS SPACE
}

\section{A. Fletcher and V. Marković}

\begin{abstract}
We present some results on the distribution of zeros of functions in the Bers space $Q(\mathbb{D})$, showing how the distribution depends on the bounds of the growth of $|f(z)|$ as $|z| \rightarrow 1$, for $f \in Q(\mathbb{D})$. We also exhibit an open and dense subset, $M \subset Q(\mathbb{D})$, which has the property of uniform control over the number of zeros in disks of hyperbolic radius 1 contained in $\mathbb{D}$.
\end{abstract}

\section{Overview}

Functions in the Bers space of the unit disk, $Q=Q(\mathbb{D})$, have restricted growth as one approaches the boundary $\partial \mathbb{D}$. We use this fact to prove some results on the distribution of the zeros of such functions in Section 2.

TheOREM 2.3. There exists a universal constant $C_{3}$ such that for all $\varphi \in Q$, there exists $r_{4} \in(0,1)$ depending on $\varphi$ such that

$$
\mathbf{n}(r) \leqslant C_{3} A(r) \log A(r), \quad\left(r \in\left(r_{4}, 1\right)\right),
$$

where we define $\mathbf{n}(r)$ to be the number of zeros of $\varphi$ in $\{z \in \mathbb{D}:|z| \leqslant r\}$ and $A(r)$ to be the hyperbolic area of this disk, for $r \in(0,1)$.

THEOREM 2.4. Let $\varphi \in Q$, then there exists an increasing sequence $\left(t_{n}\right)$ with $t_{n} \rightarrow 1$ such that $\mathbf{n}\left(t_{n}\right) \leqslant 4 /\left(1-t_{n}\right)$.

In Sections 3 and 4 , we define the subset $M \subseteq Q$ by

$$
M:=\left\{\varphi \in Q: \exists q>0, \forall z \in \mathbb{D},\|\varphi\|_{\mathbb{D}_{1}(z)}>q\right\}
$$

and prove that $M$ is open and dense in $Q$. For a given $\varphi \in M$, we have uniform control over the number of zeros on $\varphi$ in disks of hyperbolic radius 1 .

Most of the work here is needed in proving that $M$ is dense in $Q$. This is achieved using linear functionals, since the Banach dual of the space of the Bergman space of the disk, $A^{1}(\mathbb{D})$, can be identified with $Q$. We can characterize $M$ in terms of a property involving linear functionals, and work with this characterization instead.

2000 Mathematics Subject Classification: Primary 30C15; Secondary 30F30. 
A key point is that linear functionals on $A^{1}(\mathbb{D})$ corresponding to masses on small hyperbolic disks in $\mathbb{D}$ have a representation as some element of $Q$, ie. given $\Omega_{w, R}=\left\{z \in \mathbb{D}: d_{h}(z, w)<R\right\}$, define $\mu_{(w, R)}=\mathbf{1}_{\Omega_{w, R}}$ and then there exists some $\varphi_{w, R} \in Q$ such that

$$
\int_{\mathbb{D}} \mu_{(w, R)} f d x d y=\int_{\mathbb{D}} \rho^{-2} \overline{\varphi_{w, R}} f d x d y, \quad\left(\forall f \in A^{1}(\mathbb{D})\right) .
$$

This means that while we cannot add such masses to an element of $Q$ and hope to still have an element of $Q$ (let alone an analytic function), we can consider the linear functional given by adding such masses to an element of $Q$, and this will have a representation as an element of $Q$ (as hinted in the equation above). We prove the density of $M$ in $Q$ by adding appropriately small masses to elements of $Q$ which are not in $M$.

\section{Introduction}

Let $\mathbb{D}:=\{z \in \mathbb{C}:|z|<1\}$ be the open unit disk in the complex plane $\mathbb{C}$. The Bers space $Q=Q(\mathbb{D})$ is the linear normed space defined by $Q:=\{\varphi$ analytic on $\mathbb{D}$ : $\|\varphi\|<\infty\}$, where

$$
\|\varphi\|=\sup _{z \in \mathbb{D}} \frac{|\varphi(z)|}{\rho^{2}(z)}
$$

is the Bers norm, and $\rho$ is the hyperbolic density on $\mathbb{D}$, given by $\rho(z)=2 /(1-|z|)^{2}$. Let $N$ be any Riemann surface carrying the hyperbolic metric, or equivalently, has $\mathbb{D}$ as the universal cover, and let $\pi: \mathbb{D} \rightarrow N$ be the covering map. The covering group $\Gamma$ is defined by

$$
\Gamma:=\{\gamma: \mathbb{D} \rightarrow \mathbb{D} \text { conformal }: \pi(\gamma(z))=\pi(z), \forall z \in \mathbb{D}\} .
$$

The group $\Gamma$ is a discrete group of Möbius transformations on $\mathbb{D}$, and $N$ can be represented as $N \simeq \mathbb{D} / \Gamma$.

The Bers space can be generalized to any Riemann surface $N$ which can be represented as $N \simeq \mathbb{D} / \Gamma$ in the following way. If $\widetilde{\varphi}$ is a quadratic differential on $N$ (i.e., a $(2,0)$ differential form), let $\varphi$ be its lift to $\mathbb{D}$ defined by

$$
\varphi(z)=\widetilde{\varphi}(\pi(z))\left(\pi^{\prime}(z)\right)^{2}, \quad(z \in \mathbb{D}) .
$$

For any $\gamma \in \Gamma$ and $z \in \mathbb{D}$,

$$
\varphi(\gamma(z))=\widetilde{\varphi}(\pi(\gamma(z)))\left(\pi^{\prime}(\gamma(z))\right)^{2}=\widetilde{\varphi}(\pi(z))\left(\pi^{\prime}(\gamma(z))\right)^{2}\left(\gamma^{\prime}(z)\right)^{2} .
$$

The hyperbolic metric $\rho_{N}$ on $N$ is defined via the covering map $\pi$ by

$$
\rho(z)=\rho_{N}(\pi(z))\left|\pi^{\prime}(z)\right|, \quad(z \in \mathbb{D}) .
$$

For any $\gamma \in \Gamma$ and $z \in \mathbb{D}$,

$$
\rho(\gamma(z))=\rho_{N}(\pi(\gamma(z)))\left|\pi^{\prime}(\gamma(z)) \| \gamma^{\prime}(z)\right| .
$$

Combining (1.1) and (1.2) shows that the expression

$$
|\widetilde{\varphi}(\pi(z))| \rho_{N}^{-2}(\pi(z))=|\varphi(z)| \rho^{-2}(z)
$$


is invariant under change of chart, which means it is a function on the surface $N$. Thus the Bers space on $N$ is $Q(N)=Q(\Gamma):=\left\{\widetilde{\varphi}\right.$ analytic on $\left.N:\|\widetilde{\varphi}\|_{N}<\infty\right\}$, where

$$
\|\widetilde{\varphi}\|_{N}=\sup _{z \in \mathbb{D}}|\widetilde{\varphi}(\pi(z))| \rho_{N}^{-2}(\pi(z))=\sup _{z \in \mathbb{D}}|\varphi(z)| \rho^{-2}(z) .
$$

Quadratic differentials on closed Riemann surfaces are in the corresponding Bers space.

For $r \in(0,1)$, we define $\mathbf{n}(r)$ to be the number of zeros of $\varphi$ in the closed disk of radius $r$, centered at 0 . The area of the aforementioned disk will be denoted $A(r)$, and a calculation shows

$$
A(r)=\int_{\{z \in \mathbb{C}:|z| \leqslant r\}} \rho^{2}(z) d x d y=\int_{0}^{2 \pi} \int_{0}^{r} \frac{4 s}{\left(1-s^{2}\right)^{2}} d s d \theta=\frac{4 \pi r^{2}}{1-r^{2}} .
$$

Thus as $r \rightarrow 1$, we have that $A(r) \sim 1 /(1-r)$, where $f(r) \sim g(r)$ if and only if there exists a constant $\kappa \geqslant 1$ and $\xi \in(0,1)$ such that for $r>\xi$ we have

$$
\frac{1}{\kappa} \leqslant \frac{f(r)}{g(r)} \leqslant \kappa .
$$

Let $\mathbb{D}_{t}(z)=\left\{w \in \mathbb{D}: d_{h}(w, z)<t\right\}$, where $d_{h}$ denotes the hyperbolic metric. Since $\overline{\mathbb{D}_{t}(z)}$ is a compact subset of $\mathbb{D}$, any $\varphi$ which is analytic in $\mathbb{D}$ can only have finitely many zeros in $\mathbb{D}_{t}(z)$. However, as $z$ varies, the number of zeros of $\varphi$ in $\mathbb{D}_{t}(z)$ need not be bounded, even for $\varphi \in Q$, as the following example shows.

ExAmple. If $\left(\alpha_{n}\right)$ is a sequence in $\mathbb{D}$ satisfying $\alpha_{n} \neq 0$ for all $n$, and also satisfying $\sum_{n=1}^{\infty}\left(1-\left|\alpha_{n}\right|\right)<\infty$ and if $k$ is a non-negative integer, then the Blaschke product

$$
B(z)=z^{k} \prod_{n=1}^{\infty} \frac{\left(\alpha_{n}-z\right)}{\left(1-\overline{\alpha_{n}} z\right)} \frac{\left|\alpha_{n}\right|}{\alpha_{n}}, \quad(z \in \mathbb{D})
$$

is a bounded analytic function on $\mathbb{D}$, and $B$ only has zeros at $\alpha_{n}$ of order equal to the number of times the value of $\alpha_{n}$ appears in the sequence, and at the origin of order $k$. The proof of this can be found in, for example, [3]. Now let $\alpha_{n}=1-1 / n^{2}$ and $k=0$. Clearly the summation condition above is satisfied and, letting $B$ denote the Blaschke product with these zeros, $B$ is bounded and hence $B \in Q$.

$$
\beta_{n}:=\left|\frac{\alpha_{n+1}-\alpha_{n}}{1-\overline{\alpha_{n}} \alpha_{n+1}}\right|=\frac{2 n+1}{2 n^{2}+2 n}
$$

which converges to 0 as $n \rightarrow \infty$.

$$
d_{h}\left(\alpha_{n}, \alpha_{n+1}\right)=\log \left(\frac{1+\beta_{n}}{1-\beta_{n}}\right)
$$

and since $\beta_{n} \rightarrow 0$ as $n \rightarrow \infty, d_{h}\left(\alpha_{n}, \alpha_{n+1}\right) \rightarrow 0$ as $n \rightarrow \infty$. Hence the number of zeros of $B$ in $\mathbb{D}_{1}\left(\alpha_{n}\right)$ is unbounded as $n \rightarrow \infty$.

For a general analytic function on $\mathbb{D}$, the Weierstrass Factor Theorem (see [3]) says that the only condition on its zeros is that they cannot have a cluster point in $\mathbb{D}$, so $\mathbf{n}(r)$ can grow arbitrarily rapidly as $r \rightarrow 1$. However, the growth condition on Bers functions allows more to be said. 
The study of the trajectory structure of quadratic differentials is an important ingredient in the theory of quasiconformal maps and Teichmüller theory (see [5] for trajectories of quadratic differentials and, for example, [1] for the role of quadratic differentials in Teichmüller theory), as well as in the theory of harmonic maps between surfaces (see [4]). As an illustration, if $f: N_{1} \rightarrow N_{2}$ is a harmonic map between two Riemann surfaces, then the Hopf differential of $f$ is defined by $\operatorname{Hopf}(f):=\left(\rho_{N_{2}} \circ f\right) f_{z} \overline{\left(f_{\bar{z}}\right)}$. Then $f$ is quasiconformal if and only if $\operatorname{Hopf}(f) \in$ $Q\left(N_{1}\right)$.

Analyzing the extent to which one can generalize the theory of quadratic differentials to elements from the Bers space is an interesting question. The motivation for this comes from the need to understand the nature of harmonic maps, and their Hopf differentials, between non-compact hyperbolic surfaces.

The key to understanding the trajectory structure of Bers quadratic differentials is to understand the distribution of zeros. In this paper, we obtain various results on the growth of $\mathbf{n}(r)$ for $\varphi \in Q(\mathbb{D})$. The above example shows that there are $\varphi \in Q(\mathbb{D})$ for which we do not have uniform control over the number of zeros in hyperbolic disks of finite radius. However, we identify an open and dense subset of $Q(\mathbb{D})$ which has this property.

We would like to thank Mike Wolf for useful conversations related to this paper. Amongst other things, this paper deals with some questions raised by him, in particular Theorems 2.3 and 2.4 .

\section{The growth of $\mathbf{n}(r)$}

Proposition 2.1. If $\varphi \in Q$ is the lift of a quadratic differential from a compact Riemann surface $N \simeq \mathbb{D} / \Gamma$, then there exist constants $C_{0}$ and $r_{0} \in(0,1)$ depending on $\varphi$ and $\Gamma$ such that

$$
\frac{A(r)}{C_{0}} \leqslant \mathbf{n}(r) \leqslant C_{0} A(r), \quad\left(r \in\left(r_{0}, 1\right)\right),
$$

where $\mathbf{n}(r)$ and $A(r)$ are defined above.

Proof. Let $\widetilde{\varphi}$ be a quadratic differential given on a compact Riemann surface $N \simeq \mathbb{D} / \Gamma, \widetilde{\varphi} \in Q(N)$, and let $\varphi \in Q$ be its lift to $\mathbb{D}$. Let $R \subset \mathbb{D}$ be the fundamental domain for $\Gamma$ containing 0 . Since $N$ is compact, $\widetilde{\varphi}$ has only finitely many zeros, say $m$, on $N$. Hence $\varphi$ has $m$ zeros on $R$. Since $\varphi$ is the lift of a quadratic differential, it satisfies

$$
\varphi(z)=\varphi(\gamma(z))\left(\gamma^{\prime}(z)\right)^{2} \quad(\gamma \in \Gamma, z \in R) .
$$

For any $\gamma \in \Gamma$ and for all $z \in \mathbb{D}, \gamma^{\prime}(z) \neq 0$, and so for any $\gamma \in \Gamma$, the number of zeros of $\varphi$ in $\gamma(R)$ is $m$. Note that $m$ depends only on the topology of $N$ (which here in the compact case, is the genus of $N$ ).

If $p(r)$ is the number of distinct elements of $\Gamma$ which map $R$ completely inside the closed disk of radius $r$, centre at the origin, and if $q(r)$ is the number of distinct elements of $\Gamma$ which map $R$ partially inside the closed disk of radius $r$, centre at the origin, then

$$
m p(r) \leqslant \mathbf{n}(r) \leqslant m q(r) .
$$


If the hyperbolic area of $R$ is $\alpha$, then since the group $\Gamma$ is discrete, there exists $r_{0} \in(0,1)$ and constants $C_{1}, C_{2}$ depending only on $\Gamma$ such that

$$
p(r) \geqslant C_{1} A(r) / \alpha, \text { and } q(r) \leqslant C_{2} A(r) / \alpha
$$

for $r_{0}<r<1$. Combining these with (2.1) then gives the result.

To find growth conditions on $\mathbf{n}(r)$ for arbitrary $\varphi \in Q$, it is useful to consider Jensen's formula.

Proposition 2.2 (Jensen's formula). Let $\varphi$ be analytic in $\mathbb{D}, \varphi(0) \neq 0, r \in$ $(0,1)$ and $\alpha_{1}, \alpha_{2}, \ldots, \alpha_{\mathbf{n}(r)}$ the zeros of $\varphi$ listed according to multiplicities in the closed disk of radius $r$, centred at the origin. Then

$$
I(r):=\frac{1}{2 \pi} \int_{0}^{2 \pi} \log \left|\varphi\left(r e^{i \theta}\right)\right| d \theta=\log |\varphi(0)|+\sum_{i=1}^{\mathbf{n}(r)} \log \left(\frac{r}{\left|\alpha_{i}\right|}\right) .
$$

See [3] for a proof. If $\varphi \in Q$, then $|\varphi(z)| \leqslant 4\|\varphi\| /(1-|z|)^{2}$, and so

$$
I(r) \leqslant \frac{1}{2 \pi} \int_{0}^{2 \pi} \log \frac{4\|\varphi\|}{(1-r)^{2}} d \theta=\log \frac{4\|\varphi\|}{(1-r)^{2}}=: J(r) .
$$

Note that $J^{\prime}(r)=\frac{2}{1-r}$ and $A(r) \sim J^{\prime}(r)$ as $r \rightarrow 1$. Now if $\varphi$ has no zeros of modulus $r_{1}$, then since the zeros of $\varphi$ cannot have a cluster point, $\exists \delta>0$ such that $\varphi$ has no zeros on the annulus $\left\{z: r_{1}-\delta<|z|<r_{1}+\delta\right\}$ and $\mathbf{n}(r)$ is constant on the interval $\left(r_{1}-\delta, r_{1}+\delta\right)$. Jensen's formula $(2.2)$ then implies that $I$ is differentiable at $r_{1}$, with derivative $\mathbf{n}\left(r_{1}\right) / r$. However, if $\varphi$ has a zero of modulus $r_{2}$, then $\mathbf{n}(r)$ is discontinuous on every interval of the form $\left(r_{2}-\delta, r_{2}+\delta\right)$, and $I$ is not differentiable at $r_{2}$. However, defining $h(r)=\mathbf{n}(r) / r$ if $\varphi$ has no zero of modulus $r$ and $h(r)$ takes the value $\lim _{t \uparrow r} \mathbf{n}(t) / t$ otherwise, it is clear that

$$
I(r)=I\left(r_{3}\right)+\int_{r_{3}}^{r} h(t) d t
$$

and so $h$ is the distributional derivative of $I$.

THEOREM 2.3. There exists a universal constant $C_{3}$ such that for all $\varphi \in Q$, there exists $r_{4} \in(0,1)$ depending on $\varphi$ such that

$$
\mathbf{n}(r) \leqslant C_{3} A(r) \log A(r), \quad\left(r \in\left(r_{4}, 1\right)\right) .
$$

Proof. Rescaling $\varphi$ will not affect the result, so without loss of generality, we can assume $\varphi(0)=1$. If however $\varphi$ has a zero of order $k$ at 0 , then let $\widehat{\varphi}=\eta \varphi / z^{k}$, where $\eta$ is chosen so that $\widehat{\varphi}(0)=1$. The expression $|\widehat{\varphi}| \rho^{-2}=\left|\eta \varphi / z^{k}\right| \rho^{-2}$ can only possibly be unbounded at 0 since $\varphi \in Q$, but this is not the case since $\widehat{\varphi}(0)=1$, which means $\widehat{\varphi} \in Q$ and satisfies the normalization given above.

If the theorem can be proved for $\widehat{\varphi}$, then it will also hold for $\varphi$, since $\hat{\varphi}$ has a zero of order $s_{0}$ at $z_{0} \neq 0$ if and only if $\varphi$ has a zero of order $s_{0}$ at $z_{0}$. Letting $\mathbf{n}_{\psi}(r)$ denote $\mathbf{n}(r)$ for a particular $\psi \in Q$, we get $\mathbf{n}_{\widehat{\varphi}}(r)=\mathbf{n}_{\varphi}(r)-k$ for $r \in(0,1)$. Then if the theorem is true for $\widehat{\varphi}, \mathbf{n}_{\hat{\varphi}}(r) \leqslant C_{3} A(r) \log A(r)$ for $r \in\left(r_{4}, 1\right)$. Thus $\mathbf{n}_{\varphi}(r) \leqslant k+C_{3} A(r) \log A(r)$ for $r \in\left(r_{4}, 1\right)$. Since $A(r) \log A(r)$ dominates $k$ as $r \rightarrow 1$, there exists $r_{4}^{\prime} \in\left(r_{4}, 1\right)$ so that $\mathbf{n}_{\varphi}(r) \leqslant C_{3} A(r) \log A(r)$ for $r \in\left(r_{4}^{\prime}, 1\right)$. 
So, without loss of generality, $\varphi(0)=1$. Now, for a given $r \in(0,1)$, let $s \in(0,1)$ be such that $A(s)=2 A(r)$. Using (1.3),

$$
\frac{s^{2}}{1-s^{2}}=\frac{2 r^{2}}{1-r^{2}} \Rightarrow s=r \sqrt{\frac{2}{1+r^{2}}} \text {. }
$$

From (2.3),

$$
J(s)=\log \frac{4\|\varphi\|}{(1-s)^{2}} \sim \log A(s)^{2}=2 \log A(s)=2 \log 2 A(r) \sim 2 \log A(r)
$$

as $r \rightarrow 1$. Now, Jensen's formula gives

$$
I(s)=\sum_{i=1}^{\mathbf{n}(s)} \log \left(\frac{s}{\left|\alpha_{i}\right|}\right) \geqslant \sum_{i=1}^{\mathbf{n}(r)}\left(\log \left(\frac{r}{\left|\alpha_{i}\right|}\right)+\log \sqrt{\frac{2}{1+r^{2}}}\right)
$$

For $i=1, \ldots, \mathbf{n}(r)$, we have $r /\left|\alpha_{i}\right| \geqslant 1$, so

$$
I(s) \geqslant \frac{\mathbf{n}(r)}{2} \log \left(\frac{2}{1+r^{2}}\right)
$$

A Taylor expansion shows that

$$
\log \left(\frac{2}{1+r^{2}}\right) \sim 1-r
$$

as $r \rightarrow 1$. Recall that $A(r) \sim(1-r)^{-1}$ as $r \rightarrow 1$ and $I(s) \leqslant J(s)$, then combining (2.5), (2.6) and (2.7) gives $\mathbf{n}(r) \leqslant C_{3} A(r) \log A(r)$ as $r \rightarrow 1$. I.e., $\exists r_{4}$ such that for all $r \in\left(r_{4}, 1\right)$, the above holds.

The following theorem shows that the upper bound for growth given in the previous theorem cannot be maintained.

THEOREM 2.4. Let $\varphi \in Q$, then there exists an increasing sequence $\left(t_{n}\right)$ with $t_{n} \rightarrow 1$ such that

$$
\mathbf{n}\left(t_{n}\right) \leqslant \frac{4}{1-t_{n}} .
$$

Proof. Assume $\varphi(0)=1$. As in the proof of Theorem 2.3, if $\varphi$ has a zero of order $k$ at 0 , then we can replace $\varphi$ by $\widehat{\varphi}=\eta \varphi / z^{k} \in Q$ where $\eta$ is a constant chosen so that $\widehat{\varphi}(0)=1$. Then if the theorem is true for $\widehat{\varphi}$, since $\mathbf{n}_{\hat{\varphi}}(r)=\mathbf{n}_{\varphi}(r)-k$ (recall the notation given in the proof of Theorem 2.3), then $\mathbf{n}_{\varphi}\left(t_{n}\right) \leqslant k+4 /\left(1-t_{n}\right)$, and the constant $k$ term is dominated by $4 /\left(1-t_{n}\right)$ as $n \rightarrow \infty$, so can be neglected.

So without loss of generality, $\varphi(0)=1$. Assume for a contradiction, that no such sequence exists, then there exists $r_{5} \in(0,1)$ such that $\mathbf{n}(r)>4(1-r)^{-1}$ for $r \in\left(r_{5}, 1\right)$. Recall $\mathbf{n}(r)=h(r)$ almost everywhere. Then by (2.4),

$$
I(r)=I\left(r_{5}\right)+\int_{r_{5}}^{r} h(t) d t \geqslant I\left(r_{5}\right)+\int_{r_{5}}^{r} \frac{4}{1-t} d t=C_{4}\left(r_{5}\right)+2 \log \frac{4\|\varphi\|}{(1-r)^{2}}
$$

where $C_{4}$ is some constant depending only on $r_{5}$. Using the definition of $J(r)$ in (2.3) then shows that for $r$ close enough to $1, I(r)>J(r)$, which is a contradiction and gives (2.8). 
The argument in the proof shows that the constant 4 above could be replaced by any constant larger than 2 . We can say a bit more by using the above argument and that $I(r) \leqslant J(r)$.

Corollary 2.5. For $\varphi \in Q$, there does not exist $r_{6} \in(0,1)$ such that for all $r \in\left(r_{6}, 1\right)$

$$
\int_{r_{6}}^{r}\left(h(t)-J^{\prime}(t)\right) d t>0
$$

So if the uniform limit $\mathbf{n}\left(w_{n}\right)=C_{3} A\left(w_{n}\right) \log A\left(w_{n}\right)$ is achieved for a sequence $\left(w_{n}\right)$, then $h\left(w_{n}\right)-J^{\prime}\left(w_{n}\right) \rightarrow \infty$, and the corollary implies there must also be a sequence $\left(x_{n}\right)$ where $h\left(x_{n}\right)-J^{\prime}\left(x_{n}\right) \rightarrow-\infty$. Thus, in some sense, on average $\mathbf{n}(r)$ cannot exceed $J^{\prime}(r) \sim A(r)$ as $r \rightarrow 1$.

Conjecture 2.6. For $\varphi \in Q$, there exist constants $C_{5}$ and $r_{7}$ depending on $\varphi$ such that $\mathbf{n}(r) \leqslant C_{5} A(r),\left(r \in\left(r_{7}, 1\right)\right)$.

\section{Uniform control of zeros}

The example in Section 1 shows that we do not have uniform control over the zeros in disks of hyperbolic radius 1 of elements of $Q$. However, there is an open and dense subset of $Q$ where we do have uniform control over zeros. For $\varphi \in Q$, let

$$
\|\varphi\|_{\mathbb{D}_{1}(z)}:=\sup _{w \in \mathbb{D}_{1}(z)}|\varphi(w)| \rho^{-2}(w)
$$

and recall $\mathbb{D}_{1}(z)=\left\{w \in \mathbb{D}: d_{h}(w, z)<1\right\}$. Define

$$
M:=\left\{\varphi \in Q: \exists q>0, \forall z \in \mathbb{D},\|\varphi\|_{\mathbb{D}_{1}(z)}>q\right\} .
$$

Clearly $M \subset Q$. If $\varphi \notin M$, there exists a sequence $\left(z_{n}\right) \subset \mathbb{D}$ with $\left|z_{n}\right| \rightarrow 1$ such that $\|\varphi\|_{\mathbb{D}_{1}\left(z_{n}\right)} \rightarrow 0$. The aim of the rest of this article is the following theorem.

TheOREM. $M$ is an open and dense subset of $Q$.

$M$ is non-empty, since if $\widetilde{\varphi}$ is a quadratic differential on a compact Riemann surface $N \simeq \mathbb{D} / \Gamma$ and $\widetilde{\varphi} \in Q(N)$, then the lift $\varphi$ of $\widetilde{\varphi}$ to $\mathbb{D}$ is in $Q$, and satisfies $\rho^{-2}(\gamma(z))|\varphi(\gamma(z))|=\rho^{-2}(z)|\varphi(z)|$ for all $\gamma \in \Gamma$. Now, $\|\varphi\|_{\mathbb{D}_{1}(z)}>q>0$ for some $q$ and all $z$ in a fundamental region $R \subset \mathbb{D}$ of $\Gamma$. Since $\mathbb{D}=\bigcup_{\gamma \in \Gamma} \gamma(R)$, we have $\varphi \in M$.

To see $M \neq Q$, consider the little Bers space

$$
Q_{0}:=\left\{\varphi \in Q: \lim _{|z| \rightarrow 1} \rho^{-2}(z)|\varphi(z)|=0\right\} .
$$

All polynomials and functions bounded in $\mathbb{D}$, for example, are contained in $Q_{0}$. Take $\varphi \in Q_{0}$ and any sequence $\left(z_{n}\right)$ with $\left|z_{n}\right| \rightarrow 1$, then $\|\varphi\|_{\mathbb{D}_{1}\left(z_{n}\right)} \rightarrow 0$ and so $M \cap Q_{0}=\emptyset$.

Take any $\psi \in Q_{0}$ and $\varphi \in M$. Then it is clear that $\psi+\varphi \in M$. However, $(\psi+\varphi)-\varphi$ is a linear combination of two elements of $M$ which is in $Q_{0}$ and hence not in $M$. This shows that $M$ is not a linear space. 
LEMMA 3.1. Let $\left(\varphi_{n}\right)_{n=1}^{\infty}$ be any sequence of functions in $Q$ satisfying $\left\|\varphi_{n}\right\| \leqslant 1$ for all $n$. Then there exists a subsequence $\left(\varphi_{n_{i}}\right)$ which converges locally uniformly (i.e., uniformly on compact sets) to some $\varphi \in Q$ and $\|\varphi\| \leqslant 1$.

Proof. Let $K \subset \mathbb{D}$ be a compact set, and define

$$
\left\|\varphi_{n}\right\|_{K}:=\sup _{z \in K} \rho^{-2}(z)\left|\varphi_{n}(z)\right| .
$$

Now $\left\|\varphi_{n}\right\| \leqslant 1$, so $\left\|\varphi_{n}\right\|_{K} \leqslant 1$ for all $n$. This implies that for all $n$,

$$
\sup _{z \in K}\left|\varphi_{n}(z)\right| \leqslant \sup _{z \in K} \frac{4}{1-|z|^{2}},
$$

where the right-hand side is a constant depending only on $K$. This means that $\left\{\varphi_{n}\right.$ : $n=1,2, \ldots\}$ is a normal family and has a convergent subsequence $\left(\varphi_{n_{i}}\right)$, which converges locally uniformly to some analytic function $\varphi$. So $\varphi_{n_{i}} \rightarrow \varphi$ uniformly on every compact set $K$, which also means $\left\|\varphi_{n_{i}}\right\|_{K} \rightarrow\|\varphi\|_{K}$. Thus $\|\varphi\|_{K} \leqslant 1$, and since $\|\varphi\|=\sup \|\varphi\|_{K}$, where the supremum is taken over all compact sets $K$ contained in $\mathbb{D}$, then we get $\|\varphi\| \leqslant 1$.

Proposition 3.2. For any $\varphi \in M$, there exists a constant $C_{6}$ depending only on $\varphi$ such that for all $z \in \mathbb{D}$, the number of zeros of $\varphi$ in $\mathbb{D}_{1}(z)$, denoted $\mathbf{n}\left(\mathbb{D}_{1}(z)\right)$, is bounded by $C_{6}$.

Proof. Assume the result is false, then there exists a sequence $\left(z_{n}\right) \subset \mathbb{D}$ such that $\mathbf{n}\left(\mathbb{D}_{1}\left(z_{n}\right)\right)>n$. Let $A_{n}$ be the Möbius transformation $A_{n}(z)=\frac{z+z_{n}}{1+\overline{z_{n}} z}$. Then under the transformation rule $(1.1),\left(\varphi \circ A_{n}\right)\left(A_{n}^{\prime}\right)^{2} \in Q$. We also have

$$
\begin{aligned}
\left\|\left(\varphi \circ A_{n}\right)\left(A_{n}^{\prime}\right)^{2}\right\| & =\sup _{z \in \mathbb{D}} \rho^{-2}(z)\left|\varphi\left(A_{n}(z)\right)\left(A_{n}^{\prime}(z)\right)^{2}\right| \\
& =\sup _{z \in \mathbb{D}} \rho^{-2}\left(A_{n}(z)\right)\left|\varphi\left(A_{n}(z)\right)\right|=\|\varphi\|
\end{aligned}
$$

by transformation rule (1.2). So $\left\{\left(\varphi \circ A_{n}\right)\left(A_{n}^{\prime}\right)^{2}: n=1,2, \ldots\right\}$ is a sequence of elements of $Q$ with $\left\|\left(\varphi \circ A_{n}\right)\left(A_{n}^{\prime}\right)^{2}\right\|=\|\varphi\|$ for all $n$. Rescaling if necessary, Lemma 3.1 implies a subsequence $\left(\varphi \circ A_{n_{i}}\right)\left(A_{n_{i}}^{\prime}\right)^{2}$ converges locally uniformly to some $\psi \in Q$, with $\|\psi\| \leqslant\|\varphi\|$.

The number of zeros of $\left(\varphi \circ A_{n_{i}}\right)\left(A_{n_{i}}^{\prime}\right)^{2}$ on $\mathbb{D}_{1}(0)$ is unbounded as $i \rightarrow \infty$, which means the zeros of $\psi$ must have a cluster point on the compact set $\overline{\mathbb{D}_{1}(0)}$ and so $\psi$ is identically zero.

Now, $\rho^{-2}$ is bounded on $\mathbb{D}_{1}(0)$, which means, by using transformation rules (1.1) and (1.2), we have $\left|\left(\rho^{-2} \varphi\right) \circ A_{n_{i}}\right| \rightarrow 0$ uniformly on $\mathbb{D}_{1}(0)$. This is equivalent to $\|\varphi\|_{\mathbb{D}_{1}\left(z_{n_{i}}\right)} \rightarrow 0$, contradicting the fact that $\varphi \in M$.

COROllary 3.3. For any $\varphi \in M$, there exist constants $C_{7}$ and $r_{8} \in(0,1)$ depending on $\varphi$ such that $\mathbf{n}(r) \leqslant C_{7} A(r), \quad r \in\left(r_{8}, 1\right)$.

Proof. By the preceding proposition, for any $z \in \mathbb{D}$, we have $\mathbf{n}\left(\mathbb{D}_{1}(z)\right) \leqslant C_{6}$. There exists a compact Riemann surface $N \simeq \mathbb{D} / \Gamma$ such that a fundamental region $R$ is completely contained in $\mathbb{D}_{1}(0)$. As was shown in Proposition 2.1 , the number 
of distinct copies of $R$ under the covering group $\Gamma$ which are partially contained in the closed disk of euclidean radius $r$ centred at 0 grows at most linearly with $A(r)$.

In the notation of Proposition 2.1, there exists $r_{8} \in(0,1)$ such that

$$
q(r) \leqslant C_{2} A(r) / \alpha, \quad r \in\left(r_{8}, 1\right) .
$$

This means that the number of disks of hyperbolic radius 1 needed to cover the disk of euclidean radius $r$, centred at 0 , is at most $C_{2} A(r) / \alpha$, for $r \in\left(r_{8}, 1\right)$. Thus for $r \in\left(r_{8}, 1\right), \mathbf{n}(r)$ is less than the maximum number of zeros in one disk of hyperbolic radius 1 multiplied by the maximum number of such disks covering the euclidean disk of radius $r$, centred at 0 . So, $\mathbf{n}(r) \leqslant C_{6} C_{2} A(r) / \alpha, r \in\left(r_{8}, 1\right)$.

Conjecture 3.4. For any $\varphi \in M$, there exist constants $C_{7}^{\prime}$ and $r_{8}^{\prime} \in(0,1)$ depending on $\varphi$ such that $\mathbf{n}(r) \geqslant C_{7}^{\prime} A(r), r \in\left(r_{8}^{\prime}, 1\right)$.

THEOREM 3.5. $M$ is open in $Q$.

Proof. Let $\varphi \in M$ and $\|\varphi\|_{\mathbb{D}_{1}(z)}>q$ for all $z \in \mathbb{D}$. Let $\epsilon>0$ satisfy the properties $\delta:=q-\epsilon>0$, and $0 \notin \overline{\Lambda_{\epsilon}}$, where $\Lambda_{\epsilon}:=\{\psi \in Q:\|\psi-\varphi\|<\epsilon\}$. Let $\psi \in \Lambda_{\epsilon}$, then for any $z \in \mathbb{D},\|(\psi-\varphi)\|_{\mathbb{D}_{1}(z)}<\epsilon$. The triangle inequality gives

$$
\|\psi\|_{\mathbb{D}_{1}(z)}>\|\varphi\|_{\mathbb{D}_{1}(z)}-\epsilon>q-\epsilon=\delta>0 .
$$

If $q^{\prime}=\delta$, then $\|\psi\|_{\mathbb{D}_{1}(z)}>q^{\prime}>0$ for all $z \in \mathbb{D}$, hence $\psi \in M$ and so $M$ is open.

Proposition 3.6. Every element of $Q_{0}$ can be approximated by elements of $M$.

Incidentally, for any $\varphi \in Q_{0}$ and $\psi \in Q$ with

$$
\limsup _{|z| \rightarrow 1} \rho^{-2}(z)|\psi(z)|=\delta>0,
$$

then $\psi-\varphi \in Q$ and

$$
\limsup _{|z| \rightarrow 1} \rho^{-2}(z)|(\psi-\varphi)(z)|=\delta>0,
$$

which means we cannot approximate elements of $Q$ by elements of $Q_{0}$.

Proof. Let $\varphi \in Q_{0}$ and $\psi \in Q$ be the lift of a quadratic differential from a compact Riemann surface. Given any $t>0$, it will be shown that $\varphi+t \psi$ is in $M$. Now, $\rho^{-2}(z)|\varphi(z)| \rightarrow 0$ as $|z| \rightarrow 1$, so $\exists r$ such that for $|z|>r$, we have $\|\varphi\|_{\mathbb{D}_{1}(z)}<\|t \psi\|_{\mathbb{D}_{1}(z)} / 4$. Then

$$
\|(\varphi+t \psi)\|_{\mathbb{D}_{1}(z)}>\frac{\|t \psi\|_{\mathbb{P}_{1}(z)}}{2}>C_{8}>0
$$

where $C_{8}$ is some constant depending only on $t$ and $\psi$. So it is only necessary to take care of the compact subset $\{|z| \leqslant r\}$ of $\mathbb{D}$. However, $\{|z| \leqslant r\}$ is covered by finitely many $\mathbb{D}_{1}\left(z_{n}\right)$, and so $\left\{\|(\varphi+t \psi)\|_{\mathbb{D}_{1}\left(z_{n}\right)}\right\}$ is a finite set of non-negative numbers, none of which can be zero since $\varphi \neq t \psi$ (because $M \cap Q_{0}=\emptyset$ ), hence there exists a constant $q$ such that $\|(\varphi+t \psi)\|_{\mathbb{D}_{1}(z)}>q$.

So for any sequence $\left(t_{n}\right)$ converging to zero, we have $\varphi+t_{n} \psi \in M$ and $\|(\varphi+$ $\left.t_{n} \psi\right)-\varphi \| \rightarrow 0$. 
This method of proof cannot be extended to arbitrary $\varphi \in Q$, since there is the problem of cancellation when adding t $\psi$ to $\varphi$. The next section uses the methods of linear functionals to show that $M$ is indeed dense in $Q$.

\section{Linear functionals}

The Bergman space $A^{1}(\mathbb{D})$ is the Banach space of functions $f$ holomorphic in $\mathbb{D}$ satisfying

$$
\|f\|=\int_{\mathbb{D}}|f| d x d y<\infty .
$$

We have the inclusion $A^{1}(\mathbb{D}) \subset L^{1}(\mathbb{D})$, and the Hahn-Banach theorem states that any linear functional $\lambda: A^{1}(\mathbb{D}) \rightarrow \mathbb{C}$ can be extended to $\tilde{\lambda}: L^{1}(\mathbb{D}) \rightarrow \mathbb{C}$ where $\|\lambda\|=\|\widetilde{\lambda}\|$. The Riesz representation of linear functionals on $L^{1}(\mathbb{D})$ gives the existence of a unique $\mu \in L^{\infty}(\mathbb{D})$ such that

$$
\widetilde{\lambda}(f)=\int_{\mathbb{D}} \mu f d x d y
$$

for all $f \in L^{1}(\mathbb{D})$. Such a non-zero $\mu$ cannot give a zero functional on $L^{1}(\mathbb{D})$, but the restriction to $A^{1}(\mathbb{D})$ may give a zero functional.

It is known, eg from [2], that the Banach dual of $A^{1}(\mathbb{D})$ can be identified with $Q$, and for any linear functional $\lambda: A^{1}(\mathbb{D}) \rightarrow \mathbb{C}$, there exists a unique $\varphi \in Q$ such that

$$
\lambda(f)=\int_{\mathbb{D}} \rho^{-2} \bar{\varphi} f d x d y
$$

for all $f \in A^{1}(\mathbb{D})$. Denote the linear functional given by $\varphi \in Q$ by $\lambda_{\varphi}$, so

$$
\lambda_{\varphi}(f)=\int_{\mathbb{D}} \rho^{-2} \bar{\varphi} f d x d y, \quad\left(f \in A^{1}(\mathbb{D})\right) .
$$

Lemma 4.1. For any linear functional $\lambda_{\varphi}: A^{1}(\mathbb{D}) \rightarrow \mathbb{C}$, there exists a constant $C_{9}$ such that

$$
\|\varphi\| / C_{9} \leqslant\left\|\lambda_{\varphi}\right\| \leqslant\|\varphi\|, \text { where }\left\|\lambda_{\varphi}\right\|=\sup _{\|f\|=1}\left|\lambda_{\varphi}(f)\right| .
$$

Proof. Using (4.1) and (4.2),

$$
\left|\lambda_{\varphi}(f)\right| \leqslant \sup _{z \in \mathbb{D}}\left(\left|\rho^{-2}(z) \bar{\varphi}(z)\right|\right) \int_{\mathbb{D}}|f| d x d y=\|\varphi\| \int_{\mathbb{D}}|f| d x d y
$$

so $\left\|\lambda_{\varphi}\right\| \leqslant\|\varphi\|$. The proof of the other inequality can be found in [2].

Lemma 4.2. Let $\varphi_{n}, \psi \in Q,\left\|\varphi_{n}\right\| \leqslant 1$ and $\varphi_{n} \rightarrow \psi$ locally uniformly. Then for any $f \in A^{1}(\mathbb{D}), \lambda_{\varphi_{n}}(f) \rightarrow^{*} \lambda_{\psi}(f)$ in the weak-star sense.

Proof. Let $f \in A^{1}(\mathbb{D})$ and $\alpha=\int_{\mathbb{D}} \rho^{-2}|f| d x d y<\infty$. Given $\epsilon>0$, let $K \subset \mathbb{D}$ be compact and $\int_{\mathbb{D} \backslash K}|f| d x d y<\epsilon / 4$. Since $\varphi_{n} \rightarrow \psi$ locally uniformly, there exists a constant $N(K)$ such that for $n>N(K)$,

$$
\sup _{z \in K}\left|\varphi_{n}(z)-\psi(z)\right|<\frac{\epsilon}{2 \alpha} \text {. }
$$


Now for $n>N(K)$,

$$
\begin{aligned}
\mid \lambda_{\varphi_{n}}(f) & -\lambda_{\psi}(f)|=| \int_{\mathbb{D}} \rho^{-2}\left(\overline{\varphi_{n}}-\bar{\psi}\right) f d x d y \mid \\
& \leqslant \int_{K} \rho^{-2}\left|\overline{\varphi_{n}}-\bar{\psi}\left\|f\left|d x d y+\int_{\mathbb{D} \backslash K} \rho^{-2}\right| \overline{\varphi_{n}}-\bar{\psi}\right\| f\right| d x d y \\
& \leqslant\left(\sup _{z \in K}\left|\overline{\varphi_{n}}(z)-\bar{\psi}(z)\right|\right) \int_{K} \rho^{-2}|f| d x d y+\left\|\overline{\varphi_{n}}-\bar{\psi}\right\| \int_{\mathbb{D} \backslash K}|f| d x d y \\
& \leqslant \frac{\epsilon}{2 \alpha} \alpha+\left(\left\|\overline{\varphi_{n}}\right\|+\|\bar{\psi}\|\right) \frac{\epsilon}{4} \leqslant \epsilon,
\end{aligned}
$$

since $\left\|\overline{\varphi_{n}}\right\|=\left\|\varphi_{n}\right\| \leqslant 1$ and also $\|\psi\| \leqslant 1$, by Lemma 3.1 .

LEMMA 4.3. $\varphi \in M$ if and only if there is no sequence $\left(z_{n}\right) \subset \mathbb{D}$ for which $\left\|\left.\varphi\right|_{\mathbb{D}_{1}\left(z_{n}\right)}\right\| \rightarrow 0$.

Proof. Follows directly from the definition of $M$.

Define for $w \in \mathbb{D}$, the Möbius transformation

$$
A_{w}(z)=\frac{z+w}{1+\bar{w} z}
$$

Lemma 4.3 shows that if $\varphi \notin M$, then there is a sequence $\left(z_{n}\right) \subset \mathbb{D}$ such that $\|\varphi\|_{\mathbb{D}_{1}\left(z_{n}\right)} \rightarrow 0$. Since $\varphi \in Q \backslash M$, then we have $\left(\varphi \circ A_{z_{n}}\right)\left(A_{z_{n}}^{\prime}\right)^{2} \in Q$ and $\left(\varphi \circ A_{z_{n}}\right)\left(A_{z_{n}}^{\prime}\right)^{2} \rightarrow 0$ locally uniformly on $\mathbb{D}$ (recall Lemma 3.1 and the proof of Proposition 3.2). Lemma 4.2 now gives that the linear functional $\lambda_{\left(\varphi \circ A_{z_{n}}\right)\left(A_{z_{n}}^{\prime}\right)^{2}}$ converges to the zero linear functional in the weak-star sense. Let

We will now start to construct the sort of linear functionals that will be needed.

$$
\Omega_{0, R}:=\left\{z \in \mathbb{D}: d_{h}(z, 0)<R\right\}, \quad(R<\infty)
$$

denote the hyperbolic disk centred at 0 with hyperbolic radius $R$ and define

$$
\mu_{(0, R)}=\mathbf{1}_{\Omega_{0, R}},
$$

the indicator function of $\Omega_{0, R}$. By the mean value property for analytic functions, for any $f \in A^{1}(\mathbb{D})$,

$$
\int_{\mathbb{D}} \mu_{(0, R)} f d x d y=\int_{\Omega_{0, R}} \mu_{(0, R)} f d x d y=C_{10} f(0)
$$

for some constant $C_{10}$ depending on $\Omega_{0, R}$. We can clearly find $f \in A^{1}(\mathbb{D})$ such that $f(0) \neq 0$, so $\int_{\mathbb{D}} \mu_{(0, R)} f d x d y$ is a non-zero linear functional on $A^{1}(\mathbb{D})$. Hence there is a unique $\varphi_{(0, R)} \in Q$ such that

$$
\int_{\mathbb{D}} \mu_{(0, R)} f d x d y=\int_{\mathbb{D}} \rho^{-2} \overline{\varphi_{(0, R)}} f d x d y .
$$

Proposition 4.4. $\varphi_{(0, R)} \in Q_{0}$. 
Proof. For any sequence $\left(z_{n}\right)$, let $A_{z_{n}}$ be the Möbius transformations defined in (4.3). Then the sequence of functions $\left(\mu_{(0, R)} \circ A_{z_{n}}\right) \overline{A_{z_{n}}^{\prime}} / A_{z_{n}}^{\prime}$ converges locally uniformly to zero, and so the corresponding linear functionals

$$
\lambda_{n}(f)=\int_{\mathbb{D}}\left(\mu_{(0, R)} \circ A_{z_{n}}\right) \overline{\frac{A_{z_{n}}^{\prime}}{A_{z_{n}}^{\prime}}} f d x d y
$$

on $A^{1}(\mathbb{D})$ converge, in the weak-star sense, to zero. This also means that

$$
\int_{\mathbb{D}}\left(\left(\rho^{-2} \varphi_{(0, R)} \circ A_{z_{n}}\right) \frac{\overline{A_{z_{n}}^{\prime}}}{A_{z_{n}}^{\prime}}\right) f d x d y
$$

must converge weak-star to the zero linear functional as $n \rightarrow \infty$, and hence $\varphi_{(0, R)} \in$ $Q_{0}$ since $\left(z_{n}\right)$ was arbitrary.

Let $\Omega_{R}^{w}=\left\{z \in \mathbb{D}: d_{h}(z, w)<R\right\}$, for $R<\infty$ and $w \in \mathbb{D}$ (compare with (4.4)), denote the hyperbolic disk centred at $w$ with hyperbolic radius $R$, we can pullback via $A_{w}(z)$ and the pullback relation $\left(\mu_{(w, R)} \circ A_{w}\right) \overline{A_{w}^{\prime}} / A_{w}^{\prime}=\mu_{(0, R)}$ to define $\mu_{(w, R)}$ analogously to $\mu_{(0, R)}$ in (4.5) above.

Define a grid on $\mathbb{D}$ in the following way: let $S_{1}, S_{2}$, dots be concentric circles with centre at the origin and the hyperbolic radius of $S_{n}$ is $n / 10$. On each $S_{n}$ construct points $\left(t_{m}\right)$ such that $t_{0}$ is real and positive, $t_{1}$ is anticlockwise along $S_{n}$ from $t_{0}$ with $d_{h}\left(t_{0}, t_{1}\right)=1 / 10$ and continue anticlockwise around $S_{n}$ with $d_{h}\left(t_{i}, t_{i+1}\right)=1 / 10$ until $d_{h}\left(t_{m}, t_{0}\right) \leqslant 1 / 10$. Let $\left(u_{n}\right)$ denote this grid. Clearly for any $z \in \mathbb{D}$, there exist $n$ such that $d_{h}\left(u_{n}, z\right)<1$.

Theorem 4.5. $M$ is dense in $Q$.

Proof. Let $\epsilon>0$ and $\varphi \in Q$. Without loss of generality assume $\|\varphi\|=1$. Let $0<\delta<\epsilon / 10$ and define

$$
E_{\delta}:=\left\{z \in \mathbb{D}: \rho^{-2}(z)|\varphi(z)|<\delta\right\} .
$$

Recall the grid $\left(u_{n}\right)$ defined on $\mathbb{D}$. Now define linear functionals $\mu_{n}$ given by $\mu_{n}=$ $\epsilon \mu_{\left(u_{n}, 1 / 100\right)}$, if $\mathbb{D}_{1 / 100}\left(u_{n}\right) \subset E_{\delta}$ and $\mu_{n} \equiv 0$ otherwise. Then define $\mu_{\delta}=\sum_{n=1}^{\infty} \mu_{n}$. Consider the linear functional

$$
\lambda_{\epsilon}(f)=\int_{\mathbb{D}}\left(\rho^{-2} \bar{\varphi}+\mu_{\delta}\right) f d x d y .
$$

This is a non-zero linear functional on $A^{1}(\mathbb{D})$ by construction, and so there exists $\varphi_{\epsilon} \in Q$ such that

$$
\lambda_{\epsilon}(f)=\int_{\mathbb{D}} \rho^{-2} \overline{\varphi_{\epsilon}} f d x d y .
$$

We will show that $\varphi_{\epsilon} \in M$ and $\left\|\varphi_{\epsilon}-\varphi\right\|<C_{9} \epsilon$, where $C_{9}$ is the constant from Lemma 4.1, which will prove the theorem.

First assume $\varphi_{\epsilon} \notin M$, so there is a sequence of Möbius transformations $A_{z_{n}}$ associated to a sequence $\left(z_{n}\right)$ (see $\left.(4.3)\right)$ such that $\left(\rho^{-2} \varphi_{\epsilon} \circ A_{z_{n}}\right) \overline{A_{z_{n}}^{\prime}} / A_{z_{n}}^{\prime} \rightarrow 0$ 
locally uniformly. So for any $f \in A^{1}(\mathbb{D})$,

$$
\int_{\mathbb{B}_{1}(0)}\left(\left(\rho^{-2} \overline{\varphi_{\epsilon}}\right) \circ A_{z_{n}}\right) \frac{\overline{\left(A_{z_{n}}^{\prime}\right)}}{A_{z_{n}}^{\prime}} f d x d y \rightarrow^{*} 0 .
$$

Therefore $\exists N$ such that for $n>N$,

$$
\left|\int_{\mathbb{1}_{1}(0)}\left(\left(\rho^{-2} \bar{\varphi}\right) \circ A_{z_{n}}\right) \frac{\overline{A_{z_{n}}^{\prime}}}{A_{z_{n}}^{\prime}} f d x d y+\int_{\mathbb{D}_{1}(0)}\left(\mu_{\delta} \circ A_{z_{n}}\right) \frac{\overline{A_{z_{n}}^{\prime}}}{A_{z_{n}}^{\prime}} f d x d y\right|<\delta .
$$

If $\left|\left(\left(\rho^{-2} \bar{\varphi}\right) \circ A_{z_{n}}\right) \frac{\overline{A_{z_{n}}^{\prime}}}{A_{z_{n}}^{\prime}}(z)\right| \geqslant \delta$ for some $z \in \mathbb{D}_{1}(0)$, then $\left|\left(\mu_{\delta} \circ A_{z_{n}}\right) \frac{\overline{A_{z_{n}}^{\prime}}}{A_{z_{n}}^{\prime}}(z)\right|=0$ by definition, which would contradict the above inequality and this implies that $\left|\left(\left(\rho^{-2} \bar{\varphi}\right) \circ A_{z_{n}}\right) \overline{A_{z_{n}}^{\prime}} / A_{z_{n}}^{\prime}\right|<\delta$ on $\mathbb{D}_{1}(0)$. However then $\sup \left|\left(\mu_{\delta} \circ A_{z_{n}}\right) \overline{A_{z_{n}}^{\prime}} / A_{z_{n}}^{\prime}\right|=\epsilon$ on $\mathbb{D}_{1}(0)$ by the construction of $\mu_{\delta}$. So the supremum of $\mid\left(\left(\rho^{-2} \varphi+\mu_{\delta}\right) \circ A_{z_{n}}\right) \overline{A_{z_{n}}^{\prime}} / A_{z_{n}}^{\prime}$ on $\mathbb{D}_{1}(0)$ is greater than $\epsilon / 2>\delta$, which is a contradiction, so there is no such sequence $A_{z_{n}}$ with $\left(\varphi_{\epsilon} \circ A_{z_{n}}\right)\left(A_{z_{n}}^{\prime}\right)^{2} \rightarrow 0$ locally uniformly and hence $\varphi_{\epsilon} \in M$.

Now consider the linear functional

$$
\left(\lambda_{\varphi_{\epsilon}}-\lambda_{\varphi}\right)(f)=\lambda_{\left(\varphi_{\epsilon}-\varphi\right)}(f)=\int_{\mathbb{D}} \mu_{\delta} f d x d y
$$

for $f \in A^{1}(\mathbb{D})$.

$$
\left|\left(\lambda_{\varphi_{\epsilon}}-\lambda_{\varphi}\right)(f)\right| \leqslant \sup _{z \in \mathbb{D}}\left|\mu_{\delta}\right| \int_{\mathbb{D}}|f| d x d y
$$

Therefore, $\left\|\lambda_{\varphi_{\epsilon}}-\lambda_{\varphi}\right\| \leqslant \epsilon$ by the construction of $\mu_{\delta}$. Lemma 4.1 then gives $\left\|\varphi_{\epsilon}-\varphi\right\| \leqslant C_{9} \epsilon$, which completes the proof.

\section{References}

[1] O. Lehto, Univalent Functions and Teichmuller Spaces, Springer-Verlag, 1987.

[2] M. Mateljević, The dual of the Bergman space defined on a hyperbolic plane domain, Publ. Inst. Math. Nouv. Sér. 56(70) (1994) 135-139.

[3] W. Rudin, Real and Complex Analysis, McGraw-Hill, 1987.

[4] R. Schoen and S. T. Yau, Lectures on Harmonic Maps, Conf. Proc. and Lect. Not. in Geometry and Topology, Vol. II, Inter. Press, 1997.

[5] K. Strebel, Quadratic Differentials, Springer-Verlag, 1984.

Mathematics Institute University of Warwick

Coventry, CV4 7AL United Kingdom

flet cher@maths.warwick.ac.uk

markovic@maths. warwick.ac.uk
(Received 20102003 )

(Revised 2401 2004) 